\title{
Crohn's Disease with Jejunal Involvement as a Predictor of Long-Term Clinical Outcomes
}

\author{
Young Sun $\mathrm{Kim}^{1}$ and Sung-Ae Jung ${ }^{2}$ \\ ${ }^{1}$ Department of Internal Medicine, Seoul National University Hospital Healthcare System Gangnam Center, and ${ }^{2}$ Department of Internal \\ Medicine, Ewha Womans University School of Medicine, Seoul, Korea
}

See "The Clinical Characteristics and Prognosis of Crohn's Disease in Korean Patients Showing Proximal Small Bowel Involvement: Results from the CONNECT Study" by One Zoong Kim, et al. on page 67, Vol. 12. No. 1, 2018

Crohn's disease (CD) is a chronic inflammatory gastrointestinal (GI) disorder characterized by a progressive course, resulting in irreversible bowel damage. ${ }^{1}$ The ultimate goal of medical therapy in $\mathrm{CD}$ would be changing its natural disease course and preventing further progression, bowel damages and disabilities. $^{1,2}$ Therefore, it is important to understand what prognostic factors are associated with CD related complications, who is at the risk of aggressive $\mathrm{CD}$ requiring more earlier and aggressive therapies than others. ${ }^{2,3}$

Previous Western studies have shown that jejunal involvement of $\mathrm{CD}$ is associated with the higher risk of stricturing and penetrating complications, surgery, and postoperative recurrence. ${ }^{1,3}$ In addition, younger age at initial diagnosis, the presence of perianal disease, the need for systemic steroid therapies, smoking, and any significant change of serologic markers have been suggested as predictors of its aggressive disease activity. However, there only have been limited studies for these issues preformed in Korean CD patients. Moreover, CD phenotypes seem to differ considerably between East Asians and whites. ${ }^{5}$ Korean CD patients revealed a male predominance in sex distribution, predominance of ileocolonic disease in the disease location, and a higher incidence of perianal fistula in disease behavior. ${ }^{5}$ Therefore it is important to be aware of the subtypes and natural history of $\mathrm{CD}$ and the medication used to treat the disease in order to optimize the health care of patients with $\mathrm{CD}$ in different populations. ${ }^{2,5}$

In this issue of Gut and Liver, the article "The clinical characteristics and prognosis of Crohn's disease in Korean patients showing proximal small bowel involvement: results from the
CONNECT study" by Kim et al. ${ }^{6}$ performed a nation-wide cohort study including 1,329 CD patient from 32 Korean institutions. The result showed that the patients with jejunal involvement were more likely to be smokers (18.9\% vs $14.2 \%$ ), having less frequent L2 (colonic) disease (2.7\% vs 19.2\%) and fewer perianal fistulas at diagnosis (20.7\% vs $28.3 \%$ ), and displaying more complicated (stricturing) disease behavior (19.8\% vs 12.7\%) compared to those without jejunal involvement. Considering surgery-free survival of patients, the patients with jejunal involvement showed significantly lower 10 year surgery-free survival rate than those without jejunal involvement (58.4\% vs $67.8 \%, \mathrm{p}<0.001)$. After adjusting for possible confounding variables, such as age, sex, and perianal fistulas, jejunal involvement was found to be an independent risk factor for surgery after CD diagnosis (hazard ratio [HR], 1.524; 95\% confidence interval, 1.184 to 1.961$)$. In addition to jejunal involvement, female sex and complicated disease behavior were poor prognostic factors in terms of surgery-free survival (HR: female sex, 1.307; structuring behavior, 1.356; penetrating behavior, 2.131). Therefore, authors concluded that proximal small bowel involvement should be evaluated in patients with $\mathrm{CD}$ for predicting long-term clinical outcomes. They also suggested that if aggressive medical therapy is provided to patients with complicated disease behavior, also the patients with jejunal involvement should be actively considered for those treatments.

The present study is very meaningful because it is the first multicenter nation-wide cohort data providing better understandings of clinical characteristics and prognosis of Korean CD patients with jejunal involvement. The incidence of jejunal in-

Correspondence to: Young Sun Kim

Department of Internal Medicine, Seoul National University Hospital Healthcare System Gangnam Center, 152 Teheran-ro, Gangnam-gu, Seoul 06236, Korea

Tel: +82-2-2112-5632, Fax: +82-2-2112-5588, E-mail: yspanda@gmail.com

pISSN 1976-2283 eISSN 2005-1212 https://doi.org/10.5009/gnl17386

@ This is an Open Access article distributed under the terms of the Creative Commons Attribution Non-Commercial License (http://creativecommons.org/licenses/by-nc/4.0) which permits unrestricted non-commercial use, distribution, and reproduction in any medium, provided the original work is properly cited. 
volvement in the present study (16.7\%) was higher than Western countries (3.8\%), ${ }^{7}$ but was similar to the previous study performed at a single tertiary hospital in Korea (14.1\%). ${ }^{8}$ Genetic, environment factors, or difference in intestinal microbes have been suggested as possible reasons for this discrepancy. ${ }^{9}$ The inflammatory bowel disease susceptible genes that have been firmly associated with $\mathrm{CD}$ in the Caucasian population such as NOD2, interleukin-23R (IL-23R), and ATG16L1 showed no correlation with CD in Japanese, Korean, and Chinese Han patients, while IL-23R showed a weak correlation in Korean. ${ }^{9}$ However, single nucleotide polymorphisms on TNFSF15 have been identified to be more strongly associated with Asian CD patients than Caucasians. $^{9}$

In this study, upper GI involvement was more common in patients with jejunal involvement than those without (odds ratio=1.643). Authors suggested that patients with jejunal involvement should be evaluated for upper GI involvement via upper endoscopy. The prevalence of esophageal $\mathrm{CD}$ has been reported as $0.3 \%$ to $10 \%$ in adults. ${ }^{10}$ A recent prospective study have shown that the frequency of gastroduodenal CD varies geographically from $16.5 \%$ in Eastern Europeans to $2 \%$ in Asian patients. ${ }^{10}$ Upper GI involvement predicts more severe $\mathrm{CD}$ phenotypes requiring more aggressive medical and surgical intervention. ${ }^{10}$ However, only little is known about clinical characteristics and specific managements of these patients. Therefore, more studies are warranted for this issue.

Based on the result of the present study, authors suggested further study for risk-stratification of CD patients including risk factors such as complicated disease behavior and jejunal involvement to select optimal treatment strategies. Recently, another study ${ }^{2}$ result from the CONNET study conducted by the Korean Association for the Study of Intestinal Diseases (KASID) firstly validated a novel predictive model that can be used to predict the risk of surgical intervention in Korean patients with CD. As well as jejunal involvement, age at diagnosis, initial disease behavior, and perianal disease at diagnosis were associated with a poor prognosis of CD. They developed a simple point score model for CD-related surgery (simplified-the Korean Crohn's Disease Prediction, S-KCDP) based on four categorical variables (age at diagnosis $\leq 24$ years, jejunum involvement, stricturing or penetrating behavior, and perianal lesions at diagnosis). Kaplan-Meier plots stratified by the S-KCDP classification (low-risk [score 0-1], intermediate-risk [score 1.5-2], and high-risk [score $\geq 2.5$ ] groups) showed a significantly greater surgery rate in the high-risk group compared with the low-risk group (log-rank $\mathrm{p}<0.001$ ) in both the training and validation sets.
The limitation of the present study is that its cohort was established by retrospective review. Thus, this cohort was not the representative of all patients with CD in Korea. More detailed and standardized treatment protocol should be included for accurate analysis. Large scaled prospective studies including the patient's quality of life are needed to evaluate more detailed prognosis of patients having jejunal lesions, and select those who would need any early intensive treatments.

\section{CONFLICTS OF INTEREST}

No potential conflict of interest relevant to this article was reported.

\section{REFERENCES}

1. Louis E, Collard A, Oger AF, Degroote E, Aboul Nasr El Yafi FA, Belaiche J. Behaviour of Crohn's disease according to the Vienna classification: changing pattern over the course of the disease. Gut 2001;49:777-782.

2. Park Y, Cheon JH, Park YL, et al. Development of a novel predictive model for the clinical course of Crohn's disease: results from the CONNECT study. Inflamm Bowel Dis 2017;23:1071-1079.

3. Cosnes J, Cattan S, Blain A, et al. Long-term evolution of disease behavior of Crohn's disease. Inflamm Bowel Dis 2002;8:244-250.

4. Solberg IC, Cvancarova M, Vatn MH, Moum B; IBSEN Study Group. Risk matrix for prediction of advanced disease in a population-based study of patients with Crohn's disease (the IBSEN study). Inflamm Bowel Dis 2014;20:60-68.

5. Ye BD, Yang SK, Cho YK, et al. Clinical features and long-term prognosis of Crohn's disease in Korea. Scand J Gastroenterol 2010;45:1178-1185.

6. Kim OZ, Han DS, Park CH, et al. The clinical characteristics and prognosis of Crohn's disease in Korean patients showing proximal small bowel involvement: results from the CONNECT study. Gut Liver 2018;12:67-72.

7. Freeman HJ. Natural history and long-term clinical course of Crohn's disease. World J Gastroenterol 2014;20:31-36.

8. Park SK, Yang SK, Park SH, et al. Long-term prognosis of the jejunal involvement of Crohn's disease. J Clin Gastroenterol 2013;47:400-408.

9. Cheon JH. Genetics of inflammatory bowel diseases: a comparison between Western and Eastern perspectives. J Gastroenterol Hepatol 2013;28:220-226.

10. Laube R, Liu K, Schifter M, Yang JL, Suen MK, Leong RW. Oral and upper gastrointestinal Crohn's disease. J Gastroenterol Hepatol. Epub 2017 Jul 14. https://doi.org/10.1111/jgh.13866. 